Revista Arbitrada Interdisciplinaria KOINONIA

Año VI. Vol VI. N’2. Edición Especial: Educación. 2021

Hecho el depósito de Ley: FA2016000010

ISSN: 2542-3088

FUNDACIÓN KOINONIA (F.K). Santa Ana de Coro. Venezuela.

Milton Wilfrido Arévalo-Guallpa; Juan Carlos Bayas-Machado

http://dx.doi.org/10.35381/r.k.v6i2.1222

\title{
Métodos de desarrollo de habilidades motrices en la educación inicial en
} diferentes contextos educativos

Methods of developing motor skills in early education in different educational contexts

\author{
Milton Wilfrido Arévalo-Guallpa \\ milton.arevalo@est.ucacue.edu.ec \\ Universidad Católica de Cuenca, Azogues \\ Ecuador \\ https://orcid.org/0000-0003-4723-6216 \\ Juan Carlos Bayas-Machado \\ juan.bayas@ucacue.edu.ec \\ Universidad Católica de Cuenca, Azogues \\ Ecuador \\ https://orcid.org/0000-0002-5599-9240
}

Recepción: 25 de enero 2021

Revisado: 20 de febrero 2021

Aprobación: 30 de abril 2021

Publicación: 15 de mayo 2021 
Revista Arbitrada Interdisciplinaria KOINONIA

Año VI. Vol VI. N². Edición Especial: Educación. 2021

Hecho el depósito de Ley: FA2016000010

ISSN: $2542-3088$

FUNDACIÓN KOINONIA (F.K). Santa Ana de Coro. Venezuela.

Milton Wilfrido Arévalo-Guallpa; Juan Carlos Bayas-Machado

\title{
RESUMEN
}

El presente artículo de investigación se basa en la importancia que tiene la educación física en los niños que ingresan al ámbito escolar por primera vez, siendo el objetivo principal identificar los diferentes métodos que el docente puede utilizar para el desarrollo de las habilidades motrices básicas en los infantes de educación inicial. El diseño de la metodología de estudio es de tipo descriptivo, de corte transversal que permitió describir, analizar e interpretar los resultados obtenidos en la investigación. El instrumento que se utilizó fue una encuesta digital, dirigida a 110 docentes de educación inicial del distrito 01D02 Cuenca Sur. Los resultados obtenidos se pudieron evidenciar que el método más utilizado es del juego, se propone otros métodos como el cinestésico-corporal y la gamificación, mediante una guía con diferentes técnicas y actividades que ayuden al docente a mejorar las experiencias motrices básicas.

Descriptores: Educación de la primera infancia; escuela de párvulos; educación física. (Palabras tomadas del Tesauro UNESCO).

\begin{abstract}
This research article is based on the importance of physical education in children who enter school for the first time, the main objective being to identify the different methods that the teacher can use for the development of basic motor skills in students. infants of initial education. The design of the study methodology is descriptive, cross-sectional that allowed us to describe, analyze and interpret the results obtained in the research. The instrument used was a digital survey, addressed to 110 early childhood teachers from the 01D02 Cuenca Sur district. The results obtained could show that the most used method is the game, other methods are proposed such as body-kinesthetic and gamification, through a guide with different techniques and activities that help the teacher to improve basic motor experiences.
\end{abstract}

Descriptors: Early childhood education; nursery schools; physical education. (Words taken from the UNESCO Thesaurus). 
Revista Arbitrada Interdisciplinaria KOINONIA

Año VI. Vol VI. N². Edición Especial: Educación. 2021

Hecho el depósito de Ley: FA2016000010

ISSN: $2542-3088$

FUNDACIÓN KOINONIA (F.K). Santa Ana de Coro. Venezuela.

Milton Wilfrido Arévalo-Guallpa; Juan Carlos Bayas-Machado

\section{INTRODUCCIÓN}

En el proceso de enseñanza-aprendizaje motriz de los niños y niñas de educación infantil, radica el principal problema en las habilidades del equilibrio, saltos y giros, según lo expresado mediante entrevista a diferentes docentes que trabajan con estudiantes del nivel inicial, mencionando que el mayor tiempo aplican sus conocimientos dentro del aula por falta de un espacio físico para trabajar en un ambiente al aire libre.

Las estrategias metodológicas por parte del docente deben ser utilizadas de acuerdo a la edad del niño y motivarle a construir un aprendizaje significativo, entre los nuevos conocimientos y los adquiridos, que será de vital importancia en el descubrimiento de su propio cuerpo, además, le permitirá mayor integración, afectiva y social con sus pares; que el aprendizaje garantice la continuidad para el desarrollo de las habilidades motrices básicas y específicas.

La importancia de las clases de educación física debe ser considerada como una disciplina fundamental en la educación inicial; posibilita a los estudiantes desarrollar habilidades motoras, cognitivas y afectivas, expresar la espontaneidad, fomentar un aprendizaje creativo y crítico, además les permite conocer, respetar y valorarse a sí mismos, centrando su accionar en la educación del movimiento como aprendizaje que le permite vivenciar y experimentar.

En el inicio escolar es fundamental desarrollar las habilidades motrices básicas utilizando diferentes métodos con actividades motivadoras para los estudiantes, lo cual se propone realizar una guía para que los docentes tengan una mejor visión para realizar sus planificaciones. En este sentido, mediante un análisis documental en diferentes fuentes bibliográficas, se identificará los diferentes métodos que ayuden al docente en el desarrollo de habilidades motrices básicas que son fundamentales en esta edad escolar. La presente investigación tiene como objetivo identificar los métodos que son aplicables para el desarrollo de las habilidades motrices básicas en la educación inicial, el método es considerado como el camino para iniciar diferentes actividades, donde se fortalecerán las experiencias motrices que aparecen de modo innato en la evolución humana, 
Revista Arbitrada Interdisciplinaria KOINONIA

Año VI. Vol VI. N². Edición Especial: Educación. 2021

Hecho el depósito de Ley: FA2016000010

ISSN: $2542-3088$

FUNDACIÓN KOINONIA (F.K). Santa Ana de Coro. Venezuela.

Milton Wilfrido Arévalo-Guallpa; Juan Carlos Bayas-Machado

considerando como una etapa importante para su crecimiento que ayudara en el ámbito escolar, físico motriz, recreativo y cognitivo.

\section{Referencial teórico}

La educación infantil ha ido evolucionando durante el tiempo, por lo cual se considera que el docente que imparte las clases en educación inicial conozca nuevos métodos que ayuden al niño al desarrollo de habilidades motrices básicas mediante la educación física, generando que se desarrolle manera correcta. En este sentido, es necesario involucrar en la pedagogía, juegos y actividades lúdicas que contribuyan a un correcto desarrollo de las destrezas motrices de los estudiantes (Aníbal-Sailema, et al. 2017). La Didáctica es parte fundamental de la pedagogía, en la enseñanza del estudiante que se educa tanto en el medio social y cultural que lo forma, los docentes deben conocer los métodos y medios que se deben cumplir con las diferentes actividades para llegar al objetivo de la educación. Al mismo tiempo la pedagogía no puede desprenderse de la didáctica porque la educación depende de estos dos medios para llegar a un fin y la meta propuesta; la didáctica estima al juego como una estrategia de enseñanza en la educación infantil para que ellos logren desarrollar sus capacidades motrices. Además, es importante la colaboración de los padres de familia en este periodo de sus hijos e hijas, debido a que ellos son los primordiales autores de la enseñanza que reciben los pequeños antes de ingresar a los centros educativos, el desconocimiento de ellos no les posibilita contribuir con el proceso educativo primordialmente con el desarrollo de la motricidad (Razeto, 2016).

Al Método se puede definir como un procedimiento que se debe seguir para llegar a un fin por parte del docente en el proceso de enseñanza-aprendizaje, mediante la utilización de diferentes estrategias pedagógicos. Según lo expuesto por (Delgado, 1991), el método lo define como un medio de enseñanza que permite al estudiante, llegue a conseguir las metas durante el proceso educativo y sea capaz de superar diferentes retos en el futuro. En complemento, (González (2012) citado por Navarro-Lores \& Samón- 
Matos, 2017), describe el método de enseñanza como la técnica y acciones que un maestro usa con el objetivo de conseguir uno o diversos fines educativos, que tiene sentido como un todo y pueda ser compartida por la sociedad científica.

El juego-trabajo como método en el desarrollo de las habilidades motrices básicas es importante en la vida de todo ser humano y en especial en la del infante, ésta actúa como eje insustituible y fundamental en el proceso de formación integral.( Gallego-Vega, et al. 2016), por otro lado, el juego se constituye como una de las enseñanzas fundamentales en el desarrollo de las experiencias motrices y transmisor de valores propios de un pueblo o cultura, estimula al niño a un aprendizaje activo ayudando al desarrollo físico, psíquico y social (Fernández-Rivas \& Espada-Mateos, 2016).

El juego permite la acción del ejercicio, el establecimiento de normas y comportamientos al realizar la práctica, también permite desarrollar de manera integral funciones tan importantes como el tono muscular, el equilibrio, la lateralidad y las conductas perceptivo motriz, a la vez que permite adaptarse al medio físico y social (Ruiz \& Linaza, 2013). Por otro lado, el juego como estrategia de enseñanza en la educación infantil donde manifiesta que el juego es la manera natural de aprender del niño, lo cual forma su imaginación creativa, mediante la práctica contribuye al desarrollo social, afectivo de valores y normas, también es un medio ideal para la adquisición de habilidades corporales (Zepeda-Hurtado, et al. 2019).

Las inteligencias múltiples como método de enseñanza en el desarrollo de habilidades motrices básicas tenemos la inteligencia corporal-cinestésica propuesto por Howard Gardner, mediante esta inteligencia el niño puede expresar ideas y sentimientos logrando un aprendizaje significativo, además, dentro de las habilidades ayuda en el desarrollo de diferentes actividades como el atletismo, danza, arte dramático, la gimnasia, trabajos manuales y la utilización de herramientas mediante la manipulación; el docente debe generar un aprendizaje activo, también podemos destacar que las destrezas van de la mano con las habilidades y la mejor manera de fortalecer es realizando actividades al aire libre (Casanova-Zamora, et al. 2020). 
En base a la inteligencia corporal-cinestésica el niño aprende mejor manipulando, moviéndose y procesando información a través de sensaciones corporales lo que ayudaría a desarrollar las habilidades motrices básicas (Mainieri-Hidalgo, 2015). Así mismo, la inteligencia kinestésica es la capacidad de realizar diferentes actividades por parte de los estudiantes, les ayuda a resolver problemas y expresarse a través del movimiento de su cuerpo; mediante la educación física se desarrollaría las diferentes actividades lúdicas, deportivas, corporales expresivo-comunicativas y gimnásticas que beneficiarían en el desarrollo de habilidades motrices básicas (Gamboa-Jiménez, et al. 2018).

Por otro lado, la danza en la educación inicial fortalecerá el desarrollo de la habilidad motriz básica; mejorará la concentración, la coordinación, el ritmo, el equilibrio físico, la orientación y percepción espacial, además, ayudará al parvulario a conocer y rescatar costumbres y tradiciones de cada pueblo (Conesa \& Angosto, 2017). Así mismo, la gimnasia básica como actividad a tempranas edades es muy importante ya que fomenta de manera creativa la salud, la integración social, contribuye al desarrollo integral de las capacidades intelectuales, emocionales, creativos y les permite tener un aprendizaje significativo, ayuda a desarrollar de mejor manera las habilidades motrices a tener una mejor coordinación, lateralidad y equilibrio (Villarroel, et al. 2021).

En complemento, la gimnasia rítmica deportiva, aplicada al campo educativo busca desarrollar en el niño las capacidades perceptivas y motoras, la lateralidad mediante el movimiento del cuerpo, la coordinación y el perfeccionamiento de las habilidades motrices básicas y específicas (Barta-Peregot \& Duran-Delgado, 2009). En otro orden de ideas, la gamificación como método basado en los juegos de roles y mecánicas lúdicas, dentro del contexto educativo aumenta la motivación en los estudiantes, mejora las habilidades, promueve la participación activa, estimula las conexiones sociales, además generan expectativas de aprendizaje mediante el juego como elemento básico del proceso (Fernández-Gavira, et al. 2018). 
El cuento motor, jugado o vivenciado como recurso educativo define al infante como el protagonista de la clase, es estimulante y motivador a través de una narración breve, desarrolla los elementos psicomotores básicos, esquema corporal, lateralidad, estructuración espacial, temporal y ritmo, las cualidades motrices coordinativas y el equilibrio, las habilidades básicas de desplazamientos, saltos, lanzamientos, recepciones y giros (Cabrera-Valdés \& Dupeyrón-García, 2019).

Por medio de la educación física y la correcta utilización de las metodologías de enseñanza, se pretende atender las necesidades motrices de los parvularios, haciendo más fácil el desarrollo de $\mathrm{HMB}$, tanto locomotrices, no locomotrices y de manipulación, debido a que pertenecen a toda la especie humana y deben ser desarrollados de manera correcta cada una de las experiencias propias de los niños, siendo la base sobre las que se asentarán las adquisiciones motrices posteriores, más complicadas y concretas (Aníbal-Sailema, et al. 2017).

Por otro lado, el currículo de educación física, indica que las destrezas son aprendizajes básicos que se aspira impulsar en los alumnos en un área y un subnivel definido de su escolaridad que refieren a contenidos de aprendizaje, mediante movimientos en sentido extenso, destrezas o capacidades, métodos de distintos grados de dificultad, hechos, conceptos, explicaciones, reacciones, valores, de lo aprendido y desarrollado mediante la experiencia (Ministerio de educación, 2016).

Mediante los métodos y las estrategias de enseñanza se pretende que el docente fortalezca el trabajo de las habilidades motrices; en el equilibrio, siendo un estado fundamental dentro de la motricidad, cuando la persona realiza movimientos la gravedad del cuerpo se traslada y su balance depende de un equilibrio con desplazamiento y estático. Los saltos es un acto motriz donde el cuerpo se despega de la superficie, efectuando el impulso con uno o ambos pies y los giros considerados como una actividad motriz de rotación de los ejes del cuerpo humano, longitudinal, de arriba abajo, transversal, de izquierda derecha o sagital de adelante atrás. 


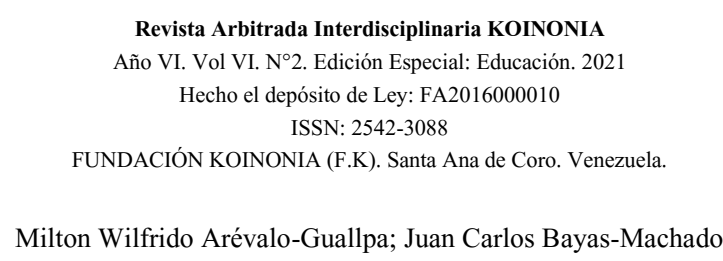

\section{MÉTODO}

La metodología de investigación que se empleó es de tipo descriptivo, de corte transversal en las que se establecen las variables que se van a estudiar a una población determinada con la recolección de datos por única vez, en base a estos resultados realizar un análisis estadístico para derivar conclusiones de la investigación (GonzálezGonzález, et al. 2020). Así mismo se apoyó en el análisis documental bibliográfica, a través de la recolección de información por medio de sitios web tales como: Google Académico, Dialnet, Scielo, donde se encontró artículos científicos relacionados con el tema y se clasificó de acuerdo a su relevancia en relación a los objetivos planteados (Prado-Prado, et al. 2020).

La población de estudio estuvo conformada por 150 docentes de educación inicial, de los cuales se realiza una muestra a 110 maestros aplicando métodos, técnicas e instrumentos de investigación. Se empleó una encuesta para diagnosticar y evaluar los estados de opinión de la muestra, realizada al personal participante en la investigación la cual consta de 10 preguntas cerradas validadas por profesionales. Se utilizó google forms para que las encuestas sean respondidas, evaluadas a través del programa Excel, en donde se insertarán todos los datos obtenidos para su posterior estudio estadístico utilizando el paquete SPSS, los mismos que ayudarán a obtener los resultados (Espinoza-Zhinin, et al. 2020).

En cuanto al análisis de los datos, se realizó por medio del programa SPSS, para procesar estadísticamente la información recopilada durante la investigación descriptiva a través de las tablas de distribución de las frecuencias absolutas (Erazo-Álvarez \& Narváez-Zurita, 2020). 


$$
\begin{gathered}
\text { Revista Arbitrada Interdisciplinaria KOINONIA } \\
\text { Año VI. Vol VI. N². Edición Especial: Educación. } 2021 \\
\text { Hecho el depósito de Ley: FA2016000010 } \\
\text { ISSN: 2542-3088 } \\
\text { FUNDACIÓN KOINONIA (F.K). Santa Ana de Coro. Venezuela. } \\
\text { Milton Wilfrido Arévalo-Guallpa; Juan Carlos Bayas-Machado }
\end{gathered}
$$

\section{RESULTADOS}

La muestra de estudio se realizó mediante encuestas a 110 docentes de Educación Inicial, a través de la plataforma Google forms, donde se puede observar los siguientes resultados.

Tabla 1. Teniendo en cuenta su experiencia como docente. ¿Cuál de estos métodos usted utiliza para el desarrollo de habilidades motrices básicas en los niños?

\begin{tabular}{lll}
\hline Opciones & Frecuencia Absoluta & Frecuencia Relativa \\
\hline Método del juego & 75 & $68,2 \%$ \\
Método de las inteligencias múltiples(corporal-cinestésico) & 25 & $22,7 \%$ \\
Método de gamificación & 10 & $9,1 \%$ \\
\hline Total & $\mathbf{1 1 0}$ & $\mathbf{1 0 0} \%$ \\
\hline
\end{tabular}

Fuente: Elaboración propia a partir de encuesta aplicada.

Análisis de la tabla 1, el muestreo establece al 68,2\% que corresponde a 75 docentes, consideran que el método del juego es el más utilizado para el desarrollo de las habilidades motrices básicas, el $22,7 \%$ que representa a 25 docentes utilizan el método de las inteligencias múltiples(corporal-cinestésico) y el 9,1\% que representa a 10 docentes utiliza el método de gamificación.

Según las encuestas, los docentes de educación inicial consideran que el juego es la mejor herramienta que ayudara al niño a mejorar y desarrollar sus experiencias motrices básicas.

Tabla 2. ¿Usted como docente planifica las actividades de acuerdo al método seleccionado para el desarrollo de habilidades motrices básicas?

\begin{tabular}{lll}
\hline Opciones & Frecuencia Absoluta & Frecuencia Relativa \\
\hline Siempre & 66 & $60 \%$ \\
Generalmente & 42 & $38,2 \%$ \\
A veces & 2 & $1,8 \%$ \\
\hline Total & $\mathbf{1 1 0}$ & $\mathbf{1 0 0 \%}$ \\
\hline
\end{tabular}

Fuente: Elaboración propia a partir de encuesta aplicada. 
Análisis de la tabla 2, el muestreo establece el $60 \%$ que corresponde a 66 docentes manifiestan que las actividades para el desarrollo de habilidades motrices básicas lo planifican de acuerdo al método seleccionado, el 38,2\% que representa a 42 docentes consideran que generalmente la planificación es de acuerdo al método y el 1,8\% que representa a 2 docentes consideran que a veces planifican sus actividades de acuerdo al método. En el estudio realizado un alto porcentaje de docentes consideran que las actividades deben ir de acuerdo al método seleccionado en el desarrollo de habilidades motrices básicas del niño en educación inicial.

Tabla 3. ¿Considera usted que el desarrollo de las habilidades motrices básicas en las clases de Educación Física es importante para el desarrollo integral de los niños?

\begin{tabular}{lll}
\hline Opciones & Frecuencia Absoluta & Frecuencia Relativa \\
\hline Siempre & 89 & $80,91 \%$ \\
Generalmente & 15 & $13,64 \%$ \\
A veces & 5 & $4,55 \%$ \\
Nunca & 1 & $0,90 \%$ \\
\hline Total & $\mathbf{1 1 0}$ & $\mathbf{1 0 0} \%$ \\
\hline
\end{tabular}

Fuente: Elaboración propia a partir de encuesta aplicada.

Análisis de la tabla 3, el muestreo establece que el 80,91\% corresponde a 89 docentes consideran que las clases de educación física son importantes y le ayudan al niño a desarrollar las habilidades motrices básicas, el 13,64\% que representa a 23 docentes manifiestan que generalmente la educación física podría ayudar al desarrollo de habilidades, el 4,5\% que representa a 5 docentes considera que a veces las clases de educación física ayudan al desarrollo de habilidades motrices y el 0,90\% que corresponde a 1 docente donde manifiesta que nunca ayuda las clases de educación física en el desarrollo de las habilidades motrices básicas. La mayoría de docentes consideran que para el desarrollo de habilidades motrices básicas en edades tempranas las clases de educación física son importantes en la educación inicial del estudiante. 
Tabla 4. ¿Considera usted que desarrollar habilidades motrices básicas en los niños ayudará a desenvolverse de mejor manera en las diferentes actividades físicas, deportivas $\mathrm{y} / \mathrm{o}$ recreativas?

\begin{tabular}{lll}
\hline Opciones & Frecuencia Absoluta & Frecuencia Relativa \\
\hline Siempre & 81 & $73,64 \%$ \\
Generalmente & 27 & $24,55 \%$ \\
A veces & 2 & $1,81 \%$ \\
\hline Total & $\mathbf{1 1 0}$ & $\mathbf{1 0 0} \%$ \\
\hline
\end{tabular}

Fuente: Elaboración propia a partir de encuesta aplicada.

Análisis de la tabla 4, el muestreo establece que el 73,64\% corresponde a 81 docentes donde consideran que el desarrollo de las habilidades motrices ayudara al niño a desenvolverse de mejor manera en las actividades físicas, deportivas y/o recreativas, el $24,55 \%$ que representa a 27 docentes consideran que generalmente las habilidades motrices ayudara al niño a desenvolverse de mejor manera en las actividades físicas, deportivas y/o recreativas y el 1,81\% que corresponde a 2 docentes manifiestan que a veces las habilidades motrices ayudan al niño a desenvolverse de mejor manera en las actividades físicas, deportivas y/o recreativas.

Mediante el análisis la mayor parte de los docentes coinciden que al realizar un buen desarrollo de habilidades en los niños tendrán un mejor desenvolvimiento en diferentes actividades física, deportivas y recreativas. 
Tabla 5. ¿Considera usted que una guía le ayudaría a tener una mejor planificación para el desarrollo de habilidades motrices básicas de los niños?

\begin{tabular}{lll}
\hline Opciones & Frecuencia Absoluta & Frecuencia Relativa \\
\hline Siempre & 79 & $71,8 \%$ \\
Generalmente & 23 & $20,9 \%$ \\
A veces & 7 & $6,4 \%$ \\
Nunca & 1 & $0,9 \%$ \\
\hline Total & $\mathbf{1 1 0}$ & $\mathbf{1 0 0} \%$ \\
\hline
\end{tabular}

Fuente: Elaboración propia a partir de encuesta aplicada.

Análisis de la tabla 5, el muestreo establece que el 71,8\% corresponde a 79 docentes creen conveniente una guía les ayudará de manera idónea en la planificación para el desarrollo de las habilidades motrices básicas, el 20,9\% que representa a 23 docentes manifiestan que habitualmente una guía les podría ayudar en su planificación, el 6,4\% que representa a 7 docentes piensan que a veces una guía puede ayudarles en la planificación y el 0,9\% que corresponde a 1 docente manifiesta que una guía no le ayudaría en su panificación para el desarrollo de habilidades motrices básicas.

Según las encuestas se puede destacar que la mayoría de participantes coinciden que una nueva guía les ayudará en sus planificaciones y así los estudiantes puedan tener un adecuado desarrollo de las habilidades motrices básicas, que necesitan ser trabajadas en edades tempranas.

\section{DISCUSIÓN}

El presente trabajo de investigación se realizó a los docentes de educación inicial pertenecientes al distrito 01D02 Cuenca Sur, según los resultados se evidencio que el método más utilizado es el juego para el desarrollo de habilidades motrices básicas durante las clases de educación física, siendo este una actividad natural que ayudará de forma relevante al desarrollo de las destrezas sociales, motrices y cognitivas, tal como se comenta en (Albornoz-Zamora, et al. 2016). 
Revista Arbitrada Interdisciplinaria KOINONIA

Año VI. Vol VI. N². Edición Especial: Educación. 2021

Hecho el depósito de Ley: FA2016000010

ISSN: 2542-3088

FUNDACIÓN KOINONIA (F.K). Santa Ana de Coro. Venezuela.

Milton Wilfrido Arévalo-Guallpa; Juan Carlos Bayas-Machado

Por otra parte, el método de las inteligencias múltiples (corporal-cinestésico) es importante para el progreso de las habilidades motrices básicas, mediante este método la docente incentiva al estudiante a que utilice su cuerpo para realizar diferentes actividades como la danza, la gimnasia básica, donde el niño podrá desarrollar de mejor manera su experiencia motriz (Ardila, 2011).

El método de la gamificación dentro de la enseñanza, ayudara al estudiante en este tiempo de pandemia, al ser una herramienta atractiva e innovadora del siglo XXI, las actividades deben ser motivadoras por parte del docente mediante una planificación adecuada tomando como referencia el cuento motor y plataformas digitales que ayudaran al desarrollo de habilidades motrices básicas en las clases de educación física y en el hogar (Fernandez-Gavira, 2018).

Es importante puntualizar que las habilidades motrices básicas aparecen de forma filogenético en la evolución humana tales como: el equilibrio, saltos y giros que el docente debe conocer para poder desarrollar en los estudiantes de manera integral durante las clases de educación física, consolidando las diferentes actividades físicas, deportivas y/o recreativas (Burgos-Angulo, et al. 2020).

Mediante la propuesta realizada, tener una guía como apoyo para los docentes de educación inicial será fundamental, donde tendrán una mejor orientación del conocimiento sobre los diferentes métodos que se utilizan para el desarrollo de HMB en la etapa de la iniciación escolar. 


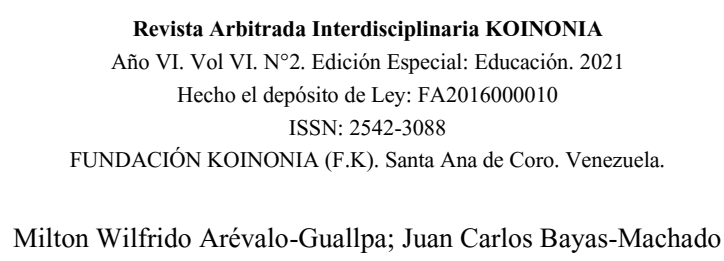

\section{PROPUESTA}

Diseño de una Guía Metodológica para el desarrollo de habilidades motrices básicas orientada a los estudiantes de educación inicial en las clases de Educación Física.

\section{Fundamentación teórica de la propuesta}

Es la información recopilada sobre el tema de estudio que servirá para ejecutar la guía como propuesta para los docentes de educación inicial, sobre los diferentes métodos de enseñanza en el desarrollo de habilidades motrices básicas; entre ello tenemos: el método del juego, inteligencias múltiples (cinestésico-corporal), y la gamificación. Estas metodologías ayudaran a los niños en las clases de educación física, a realizar diferentes actividades y ejercicios, a través del juego (tradicionales y nuevos), la danza, la dramatización del cuento, la gimnasia básica y la tecnología (TIC).

Mediante esta guía el docente podrá conocer cómo influye los métodos y las actividades, de manera positiva o negativa en el desarrollo de las habilidades motrices básicas en los niños de educación inicial y les ayudará a desenvolverse de manera correcta y progresiva.

\section{Objetivo general de la propuesta}

Proponer una guía basada en los diferentes métodos para el desarrollo de las habilidades motrices básicas y alcanzar una participación activa en el proceso de enseñanza aprendizaje.

\section{Objetivos específicos}

Definir las actividades con los diferentes métodos que existen para el desarrollo de las habilidades motrices básicas en edades tempranas mediante la investigación científica. Aplicar los métodos con las actividades más importantes para el mejoramiento de las habilidades motrices básicas. 


\section{Guía metodológica}

Objetivo: Mediante el juego de la rayuela los niños mejoraran el desarrollo de sus habilidades motrices básicas.

\begin{tabular}{|l|l|l|}
\hline Método: Juego & Recursos: Tiza, tejo & $\begin{array}{l}\text { Beneficios: Mejora la } \\
\text { coordinación, el equilibrio, } \\
\text { saltos, giros y lateralidad. }\end{array}$ \\
\hline
\end{tabular}

Desarrollo: Dibujar un diagrama compuesto por 10 cuadros, luego escribe el número del 1 al 10 con diferentes colores de tiza. La ubicación del niño, debe estar detrás del primer cuadro, lanza la piedra, donde caiga, el estudiante no podrá pisar, el estudiante comenzara el circuito con un salto a la pata coja si existe un cuadro o dos pies si el cuadrado el doble. Si el niño cae o la piedra sale del cuadrado, tiene que ceder el turno al siguiente jugador.

Otro tipo de actividad que ayudará en el desarrollo de las habilidades motrices básicas, es la rayuela africana, que no es considerado un juego competitivo, su diagrama puede ser de $4 \times 4$ cuadrados del mismo tamaño, mediante una coreografía simétricamente de saltos y giros, manteniendo el equilibrio en cada uno de los pasos. El número de participantes puede ser (1, 2, 3 o 4 o más) acompañada de una canción "Amarelinha africana". 
Objetivo: Incentivar al conocimiento de las tradiciones del Ecuador, mediante la danza que permitirá el desarrollo de las habilidades motrices básicas a través de la expresión corporal.

\begin{tabular}{|l|l|l|}
\hline Método: $\quad$ Cinestésico- & Recursos: música & $\begin{array}{l}\text { Beneficios: Ayuda en el } \\
\text { desarrollo intelectual y } \\
\text { corporal }\end{array}$ \\
motriz, su movimiento es \\
vivo tiene semejanza con el \\
pasodoble o la marcha.
\end{tabular}

Desarrollo: El baile del pasacalle proviene de la palabra francesa "passacaille", este es un ritmo mestizo que dio sus primeros indicios en la costa ecuatoriana, que al pasar de los tiempos mediante los comerciantes fue conocido en nuestra sierra ecuatoriana, el mismo se lo practica como su nombre lo dice pasar la calle en diferentes eventos culturales de la urbe, su movimiento se destaca por tener 4/4 en tiempo binario en el cual nos permite llevar correctamente su baile y el compás corporal.

Este ritmo se lo zapatea con los dos pies de una forma suave acompañada con un perfecto balanceo de rodillas y caderas, la posición de los brazos tanto del hombre como de la mujer puede variar de acuerdo al sector y atuendo tradicional de cada provincia.

Objetivo: Motivar, mediante la tecnología a que realicen actividad física, que les ayudará en el desarrollo de habilidades motrices básicas.

\begin{tabular}{|l|l|l|}
\hline Método: Gamificación & Recursos: Tablet, & Metodología: mediante la \\
& computadora, proyector. & tecnología, realizar \\
& actividades físicas con \\
\hline
\end{tabular}




\begin{tabular}{|l|l|l|}
\hline & & $\begin{array}{l}\text { diferentes plataformas } \\
\text { virtuales. }\end{array}$ \\
\hline Desarrollo: GoNoodle es una plataforma que ofrece videos gratuitos y de pago, que \\
tienen como propósito entretener a los niños y hacerlos mover al mismo tiempo. Ponen \\
a niños corriendo al mediante la realidad virtual o siguiendo pasos de baile.
\end{tabular}

\section{CONCLUSIONES}

En este trabajo de investigación, se identificaron diferentes métodos de enseñanzaaprendizaje que ayudarán a los docentes de educación inicial en las clases de Educación Física, los mismos que fueron investigados en varias fuentes bibliográficas. Además, se realizó una encuesta dirigida a los docentes, quienes son los que trabajan de manera directa con los estudiantes y conocen las dificultades que tienen los niños en el desarrollo de las habilidades motrices básicas.

El profesor debe contar con una variedad de métodos como el juego, cinestésico-corporal y la gamificación con varias actividades de enseñanza, que favorezcan al aprendizaje significativo del estudiante y correcto progreso motriz, Además, de contar con una guía que favorecerá en el desarrollo de todas las habilidades locomotrices, no locomotrices y de manipulación, mediante las cuales el estudiante podrá desenvolverse de manera correcta en cada una de sus habilidades motrices en las diferentes etapas de su crecimiento durante el periodo escolar. 
Revista Arbitrada Interdisciplinaria KOINONIA

Año VI. Vol VI. N². Edición Especial: Educación. 2021

Hecho el depósito de Ley: FA2016000010

ISSN: 2542-3088

FUNDACIÓN KOINONIA (F.K). Santa Ana de Coro. Venezuela.

Milton Wilfrido Arévalo-Guallpa; Juan Carlos Bayas-Machado

\section{FINANCIAMIENTO}

No monetario.

\section{AGRADECIMIENTO}

A la Universidad Católica de Cuenca y la Jefatura de Posgrado; por su apoyo a la investigación formativa.

\section{REFERENCIAS CONSULTADAS}

Albornoz-Zamora, E, \& del-Carmen-Guzmán, M. (2016). Desarrollo cognitivo mediante estimulación en niños de 3 años: centro desarrollo infantil nuevos horizontes. Quito, Ecuador [Cognitive development through stimulation in 3 year-old children: center for child development "nuevos horizontes". Quito, Ecuador]. Revista Universidad y Sociedad, 8(4), 186-192.

Aníbal-Sailema, Á, Sailema-Torres, M, Amores-Guevara, P, Navas-Franco, L, MallquiQuisintuña, V, \& Romero-Frómeta, E. (2017). Juegos tradicionales como estimulador motriz en niños con síndrome de Down [Traditional games as motor stimulator in children whit Down syndrome]. Revista Cubana de Investigaciones Biomédicas, 36(2), 1-11.

Ardila, R. (2011). Inteligencia. ¿qué sabemos y qué nos falta por investigar? [Intelligence. What do we know and what are we missing to investigate?]. Revista de la Academia Colombiana de Ciencias Exactas, Físicas y Naturales, 35(134), 97-103.

Barta-Peregot, A, \& Duran-Delgado, C. (2009). Mil ejercicios y juegos de gimnasia rítmica deportiva [A thousand exercises and games of sports rhythmic gymnastics]. Recuperado de https://n9.cl/i2jpg

Burgos-Angulo, D. J., Rendón-Lainez, V. M., Díaz-Nivela, C. de L., Aguirre-Chiriguayo, J. T., Peralta-Zuñiga, G. A., \& Angulo-de-León, T. A. (2020). La relajación y estrés laboral en el personal docente: una revisión sistemática [Relaxation and work stress in teaching staff: a systematic review]. Lecturas: Educación Física $Y$ Deportes, 25(266), 109-123. https://doi.org/10.46642/efd.v25i266.1617 
Revista Arbitrada Interdisciplinaria KOINONIA

Año VI. Vol VI. N². Edición Especial: Educación. 2021

Hecho el depósito de Ley: FA2016000010

ISSN: 2542-3088

FUNDACIÓN KOINONIA (F.K). Santa Ana de Coro. Venezuela.

Milton Wilfrido Arévalo-Guallpa; Juan Carlos Bayas-Machado

Cabrera-Valdés, B, \& Dupeyrón-García, M. (2019). El desarrollo de la motricidad fina en los niños y niñas del grado preescolar [The development and of fine motor skills in pre-school children]. Mendive. Revista de Educación, 17(2), 222-239.

Casanova-Zamora, T. A., Navas-Bonilla, C. del R., Piñas-Morales, M. B., \& VásquezCáceres, M. G. (2020). La educación superior ecuatoriana en el campo investigativo. Destrezas y sociedad [Ecuadorian higher education in the research field. Skills and society]. Revista Boletín Redipe, 9(9), 65-75. https://doi.org/10.36260/rbr.v9i9.1063

Conesa, E., \& Angosto, S. (2017). La expresión corporal y danza en la educación física de secundaria y bachillerato [Body expression and dance in physical education of secondary and high school]. Cuadernos de Psicología del Deporte, 17(2), 111120.

Delgado, M.A. (1991). Hacia una clarificación conceptual de los términos en didáctica de la educación física y el deporte [Towards a conceptual clarification of the terms in didactics of physical education and sport]. Revista de educación física, 40, 2-10.

Erazo-Álvarez, J., \& Narváez-Zurita, C. (2020). Medición y gestión del capital intelectual en la industria del cuero - calzado en Ecuador. [Measurement and management of intellectual capital in the leather industry - footwear in Ecuador] Revista Arbitrada Interdisciplinaria Koinonía, 5(9), 437-467. http://dx.doi.org/10.35381/r.k.v5i9.662

Espinoza-Zhinin, J., García-Herrera, D., Álvarez-Lozano, M., \& Erazo-Álvarez, J. (2020). Estrategias tecnológicas para fomentar las concepciones y su rol en los docentes de nivel inicial [Technological strategies to promote conceptions and their role in initial level teachers]. Revista Arbitrada Interdisciplinaria Koinonía, 5(5), 50-67. http://dx.doi.org/10.35381/r.k.v5i5.1033

Fernández-Gavira, J, Prieto-Gallego, E, Alcaraz-Rodríguez, V, Sánchez-Oliver, A, Grimaldi-Puyana, M. (2018). Aprendizajes Significativos mediante la Gamificación a partir del Juego de Rol: "Las Aldeas de la Historia" [Significant Learning through Gamification from Role Play: "The Villages of History"]. Revista Digital "Espiral. Cuadernos del Profesorado", 11(22), 69-78. http://dx.doi.org/10.25115/ecp.v11i21.1919 
Revista Arbitrada Interdisciplinaria KOINONIA

Año VI. Vol VI. N². Edición Especial: Educación. 2021

Hecho el depósito de Ley: FA2016000010

ISSN: 2542-3088

FUNDACIÓN KOINONIA (F.K). Santa Ana de Coro. Venezuela.

Milton Wilfrido Arévalo-Guallpa; Juan Carlos Bayas-Machado

Fernández-Rivas, M., \& Espada-Mateos, M. (2016). Formación inicial y percepción del profesorado sobre los estilos de enseñanza en Educación Física [Teachers' education and perception of teaching styles in Physical Education]. Retos, 31, 6975. https://doi.org/10.47197/retos.v0i31.49024

Gallego-Vega, C., Rodríguez-Gallego, M. R., \& Corujo-Vélez, C. (2016). La perspectiva comunitaria en la educación inclusiva [The community perspective in inclusive education]. Revista Prisma Social, (16), 60-110.

Gamboa-Jiménez, R, Jiménez-Alvarado, G, Peña-González, N, Gaete-Navarro, C, \& Aguilera-Ubeda, D. (2018). Prácticas corporales e innovación en educación infantil (0-6 años):

análisis crítico desde la mirada de expertos [Body practices and innovation in early childhood education (0-6 years): critical analysis from the point of view of experts]. Revista Brasileira de Ciências do Esporte, 40(3),224-232.

González-González, D., García-Herrera, D., Cabrera-Berrezueta, L., \& Erazo-Álvarez, J. (2020). Herramientas tecnológicas aplicadas por los docentes durante la emergencia sanitaria COVID-19 [Technological tools applied by teachers during the COVID-19 health emergency]. Revista Arbitrada Interdisciplinaria Koinonía, 5(1), 332-350. http://dx.doi.org/10.35381/r.k.v5i1.786

Mainieri-Hidalgo, A. (2015). Conocimientos Teóricos y Estrategias Metodológicas que Emplean Docentes de Primer Ciclo en la Estimulación de las Inteligencias Múltiples [Theoretical Conceptualizations and Methodological Strategies used by First Cycle Teachers in Implementing the Theory of Multiple Intelligences]. Actualidades Investigativas en Educación, 15(1), 147-186.

Ministerio de educación (2016). Currículo de educación física [Physical Education Curriculum]. Recuperado de https://n9.cl/zh02

Navarro-Lores, D, \& Samón-Matos, M. (2017). Redefinición de los conceptos método de enseñanza y método de aprendizaje [Redefinition of the concepts of teaching method and learning method]. EduSol, 17(60), 26-33.

Prado-Prado, S., García-Herrera, D., Erazo-Álvarez, J., \& Narváez-Zurita, C. (2020). Google Classroom: educational application as a learning environment in rural areas in contexts of COVID-19. Revista Arbitrada Interdisciplinaria Koinonía, 5(5), 4-26. http://dx.doi.org/10.35381/r.k.v5i5.1031 
Revista Arbitrada Interdisciplinaria KOINONIA

Año VI. Vol VI. N². Edición Especial: Educación. 2021

Hecho el depósito de Ley: FA2016000010

ISSN: 2542-3088

FUNDACIÓN KOINONIA (F.K). Santa Ana de Coro. Venezuela.

Milton Wilfrido Arévalo-Guallpa; Juan Carlos Bayas-Machado

Razeto, A. (2016). El involucramiento de las familias en la educación de los niños: Cuatro reflexiones para fortalecer la relación entre familias y escuelas [The parent involvement in the education of the children. Four reflections to strengthen the relation between families and schools]. Páginas de Educación, 9(2), 184-201.

Ruiz, L, \& Linaza, J. (2013). Jerome Bruner y la organización de las habilidades motrices en la infancia [Jerome Bruner and the organization of motor skills in childhood]. RICYDE. Revista Internacional de Ciencias del Deporte, IX(34),390-395.

Villarroel, R., Santa María, H., Quispe, V., \& Ventosilla, D. (2021). La gamificación como respuesta desafiante para motivar las clases en educación secundaria en el contexto de COVID-19 [Gamification as a challenging response to motivate classes in secondary education in the context of COVID-19]. Revista Innova Educación, 3(1), 6-19. https://doi.org/10.35622/j.rie.2021.01.001

Zepeda-Hurtado, M, Cardoso-Espinosa, E \& Rey-Benguría, C. (2019). El desarrollo de habilidades blandas en la formación de ingenieros [The development of soft skills in the training of engineers]. Científica, 23(1),61-67.

(C2021 por los autores. Este artículo es de acceso abierto y distribuido según los términos y condiciones de la licencia Creative Commons Atribución-NoComercial-Compartirlgual 4.0 Internacional (CC BY-NC-SA 4.0)

(https://creativecommons.org/licenses/by-nc-sa/4.0/). 